\title{
New born hearing screening programme at tertiary care hospital from South Karnataka-our experience
}

\author{
Manjunath V.G. ${ }^{1}$, Reddy S.K. ${ }^{2}$, K. Jagadish Kumar ${ }^{3}$ \\ ${ }^{1}$ Dr. V.G. Manjunath, Associate Professor, ${ }^{2}$ Dr. Shravan Krishna Reddy, Resident Post Graduate, ${ }^{3}$ Dr. K. Jagadish \\ Kumar, Professor, Pediatrics, all authors are affiliated with Department of Pediatrics, JSS Medical College, JSS \\ Academy of Higher Education \& Research (JSS AHER), Mysore, Karnataka, India.
}

Corresponding Author: Dr. V.G. Manjunath, Associate Professor of Pediatrics, JSS Medical College, JSS AHER, Mysore, India. Email: vghunsur@rediffmail.com

\begin{abstract}
Background: Universal hearing screening is implemented in many developed countries. However, neither universal screening, nor high risk screening, exists uniformly all over India. Screening of only high risk neonates can miss upto $50 \%$ of babies with hearing loss, hence a cost effective universal screening programme will be the viable option. Objective: To determine the incidence of hearing impairment in normal newborns with no risk factors delivered in a tertiary hospital in south Karnataka, India. Methods: All eligible newborns were screened using two staged Transient Evoked Oto Acoustic Emissions (TEOAE) at birth and at 4-6 weeks of age and confirmatory test by auditory brainstem response (ABR) at 3 months of life. Results: Out of 977 babies, 14 were detected with hearing loss at first follow up at 4 6 weeks of age. Of these 14 babies, 6 were confirmed to have hearing impairment using ABR at 3 months of age. Two of them had severe sensory neural hearing loss and the other four were diagnosed with moderate to severe hearing loss. The incidence of hearing loss in our study is 6.1 per 1000 neonates with no risk. Conclusions: A two-staged TEOAE hearing screening can be feasible option as newborn hearing screening method, for early detection of hearing impairment in all major hospitals. Universal hearing screening is the need of the hour to detect large number of hearing impaired in the magnanimous "no risk" newborn population in our country.
\end{abstract}

Key words: Newborn, Hearing Screening, No Risk, Transient Evoked Oto Acoustic Emissions

\section{Introduction}

Congenital, bilateral hearing impairment occurs in approximately $1-5$ per 1000 live births and permanent unilateral hearing loss is also included, the incidence increases up to 8 per 1000 live births [1-3]. Hearing impairment is usually detected after 2 years, by which time there is irreversible damage to the language development potential of the child. [4]. Early identification and intervention for hearing loss by 6 months of age provides better prognosis in language development, academic success, social integration and successful participation in the society [3].

Different studies have revealed Transient Evoked Oto Acoustic Emissions (TEOAE) sensitivity as high as $95 \%-98 \%$ and a specificity of $80 \%-85 \%$ [5,6]. TEOAE was preferred as screening tool as it is cost effective, convenient, easy to use and timesaving. ABR was used

Manuscript received: $28^{\text {th }}$ September 2018

Reviewed: $7^{\text {th }}$ October 2018

Author Corrected: $15^{\text {th }}$ October 2018

Accepted for Publication: $20^{\text {th }}$ October 2018 to confirm the hearing defect in TEOAE failed infants to decrease the false alarm and unnecessary intervention. Two staged TEOAE followed by confirmation by auditory brainstem response (ABR), as per the recommendations of National Institutes of Health Consensus (NIHC) Development Conference Statement was used to diagnose hearing impairment [7]. Hence this study was undertaken with the objective of documenting the burden of hearing impairment in newborns with no risk factors delivered at tertiary care hospital with the aim of early diagnosis and intervention. We have tried to look into the incidence of hearing impairment in no risk group using two staged TEOAE with confirmatory ABR.

\section{Material and Methods}

This prospective study was conducted at our tertiary care teaching hospital after obtaining institutional research and ethical committee approval. 
Study design: Prospective observational study.

Place of study: This was done at JSS Medical College Hospital, Mysore, Karnataka, India

Study period: The study was conducted during the period starting from $1^{\text {st }}$ August 2013 to 31st August 2014.

Subjects and sample size: All newborns delivered during the study period were enrolled for the study after obtaining prior informed consent form either parents or caretakers. All enrolled babies were screened for risk factors included in the High Risk Registry (HRR) of Joint Committee on Infant Hearing (JCIH) in 2007 [8]. All those babies who had no risk factors were included for the study. Babies with any one or more of the risk factors were excluded.

The Risk indicators included-Family history of permanent childhood hearing loss, Neonatal intensive care of more than 5 days or any of the following regardless of length of stay: Extracorporeal Membrane Oxygenation (ECMO) therapy, assisted ventilation, exposure to ototoxic medications or loop diuretics and hyper bilirubinemia that requires exchange transfusion. In utero infections, such as Cytomegalovirus (CMV), herpes, rubella, syphilis etc. Craniofacial anomalies, including those that involve the pinna, ear canal, ear tags, ear pits, and temporal bone anomalies.
Original Research Article

Study protocol: The study protocol was carried out in three steps [Screening/Re-screening Protocol - as per flow chart -1].

1. Initial Screening: All no risk newborns in the study were screened by TEOAE between 48-72 hours of life. This screening was conducted in noiseless room with babies in sleeping state after confirmation of nonobstructive external auditory canal by the pediatric resident. Those babies who failed the initial screening were called for follow up at 4-6weeks of age.

2. First follow- up screening- was done at 4 to 6 weeks of age by TEOAE for no risk babies who failed the first test screening ('refer category). Both first and second screening was done using a GSI Audio Screener SN20008P ${ }^{\mathrm{TM}}$, which is a completely automated analysis system that gives a "PASS" or "REFER" result. Absence of emissions for 2 out of the 3 frequencies tested ( $2 \mathrm{kHz}, 3 \mathrm{kHz}$ and $4 \mathrm{kHz}$ ) was given a "REFER" result. Infants who failed the screening twice were called for second follow up at 3 months of age.

3. Second follow- up screening was done at 3 months age to confirm the hearing impairment by ABR test. Those confirmed to have hearing impairment were referred to an audiologist for further management.

Statistical analysis: The data collected was compiled and analysis done using Microsoft excel.

\section{Results}

A total of 1400 deliveries were conducted in this one-year study period that included both vaginal delivery and the LSCS. Based on the risk indicators, 423 neonates were excluded from the study.

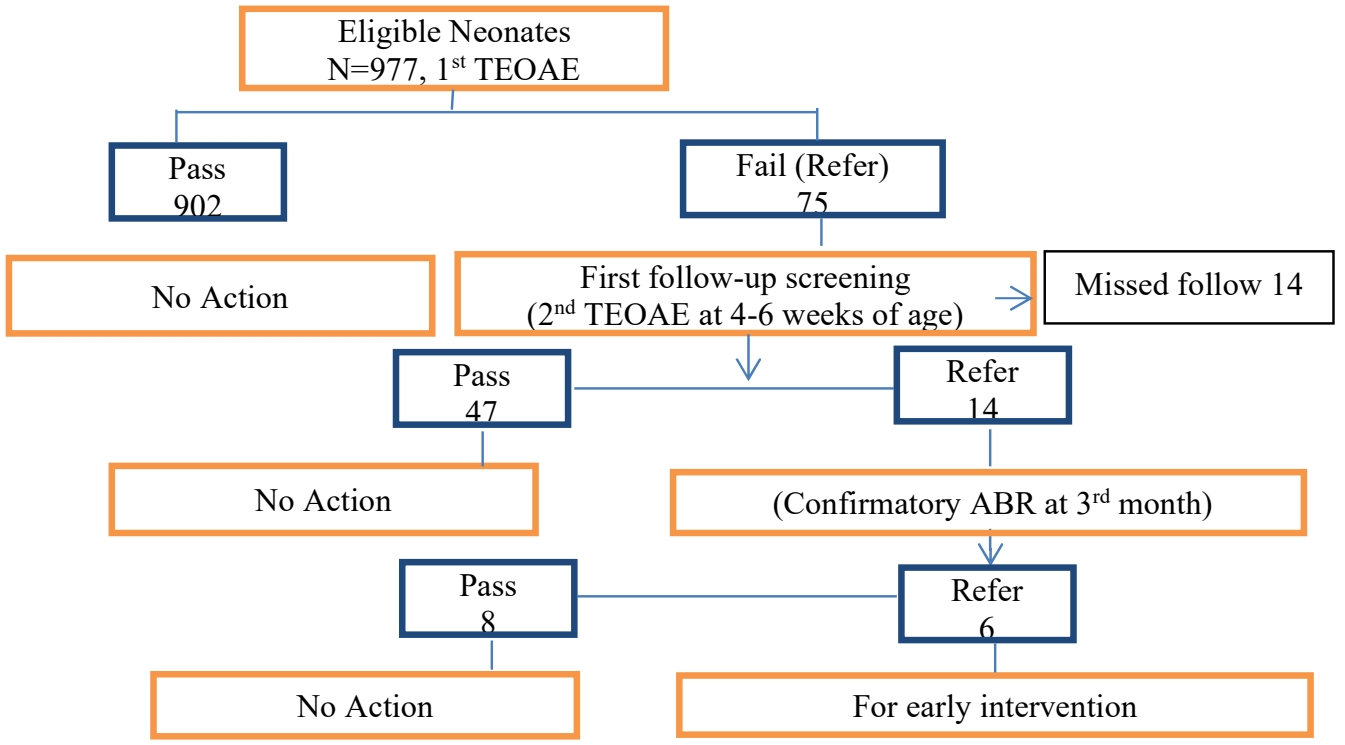

Fig-1: Flow chart depicting Screening Protocol and outcome 
Among the 977 neonates with no risk factors who were screened initially, seventy five (75) babies failed the first screening. Out of the 75 neonates who failed the first screening, 61 babies came for follow up between 4 to 6 weeks of age, however 14 babies did not turn up for follow up in spite of repeated reminders. Among those 61 babies who attended the second screening 14 babies failed in the second screening as well. All these 14 babies with no risk factors who failed the second screening were tested for hearing loss by ABR at 3 months of age. Six babies out of the above 14 were confirmed to have hearing loss. Two of them had severe sensorineural hearing loss and the other four were diagnosed with moderate to severe hearing loss.

Table-1: Demographic details of newborns screened and outcome.

\begin{tabular}{|c|c|c|}
\hline \multicolumn{2}{|c|}{ Total new borns screened $\mathbf{n}=977$} & Hearing loss confirmed $n=6$ \\
\hline \multicolumn{3}{|c|}{ Gender } \\
\hline Male & $519(53.1)$ & 3 \\
\hline Female & $458(46.9)$ & 3 \\
\hline \multicolumn{3}{|c|}{ Birth weight in kg } \\
\hline$<2.5$ & $139(14.2)$ & 1 \\
\hline $2.5-3.5$ & $770(78.8)$ & 5 \\
\hline$>3.5$ & $68(7)$ & 0 \\
\hline \multicolumn{3}{|c|}{ Mode of Delivery } \\
\hline LSCS & $411(42.1)$ & 4 \\
\hline Normal & $566(57.9)$ & 2 \\
\hline
\end{tabular}

Out of 977 newborns that were screened, males constituted $53.1 \%$ while females were $46.9 \%$. Majority of the newborns (78.8\%) who were screened weighed between $2.5-3.5 \mathrm{~kg}$, while babies below $2.5 \mathrm{~kg}$ constituted $14.2 \%$ and $7 \%$ were above $3.5 \mathrm{~kg}$. Mode of delivery was normal in $57.9 \%$ and LSCS in $42.1 \%$ of the newborns screened. Out of 566 who were delivered normally 2 were confirmed to have hearing loss. Among 411 babies delivered by LSCS, 4 newborns had hearing loss. All the 6 babies with hearing loss were referred to an audiologist for further management.

\section{Discussion}

Early identification of hearing loss with appropriate interventions will minimize developmental delays and facilitate communication, education and social development [9]. Congenital hearing loss can be detected within the first few days after birth by newborn screening [9]. It is well documented that targeted screening of high risk infants only would miss $50 \%$ of babies that would have been identified by universal new born hearing screering [UNHS][10].

However, there are limitations to this universal hearing screening. This will not identify progressive and late onset hearing loss as well as less severe hearing loss [11]. Research suggests that children who are born deaf or acquire hearing loss very early in life and who receive appropriate interventions within six months of age are at par with their hearing peers in terms of language development by the time they are five years old (in the absence of other impairments) [9]. Without a standard screening programme hearing loss is not detected till 24 months of age when it is identified with language delay. Universal screening has reduced the age at which infant receives hearing aid to 5 to 6 months from 13 to 16 months [12]. In a study by Rai et al, out of 439 neonates in "no risk" group screened, males constituted $52.84 \%$ and females $47.16 \%$ which is similar to our study [13]. They also observed that $60.5 \%$ of babies weighed more than $2.5 \mathrm{kgs}$ at birth where as in our study $85 \%$ of the babies were more than $2.5 \mathrm{~kg}$ [13]. In the present study, out of 977 screened, 75 failed the initial TEOAE test, accounting to a referral rate of $7.67 \%$ which is similar $(7.72 \%)$ to a study done by Sangita et al [14]. It's worth while to note that among the 15 hearing impaired detected in their study 7 did not have any risk factor, again emphasizing the need of UNHS as the ideal strategy of hearing screening for neonate [14].

In our study out of the 977 infants who had "no risk "of hearing, 6 infants were detected to have hearing loss. Therefore, the frequency of hearing loss in our study is 6.14 per 1000 . Hence just an "at risk" hearing screening would have missed detection of these infants. Although literature suggests that the incidence of hearing impaired in "no risk" group is much less than the incidence in the "at risk" group, the magnanimity of newborn population in "no risk" group in our country is huge, leading to a large number hearing impaired 
missed by only high-risk screening. The fact that out of 75 babies who failed the first screening test, 61 came for second screening leading to follow up rate of $81.33 \%$ also supports the feasibility of two staged TEOAE. However in a study by Augustine et al only $35.6 \%$ came for follow-up[15]. Congenital, bilateral hearing impairment occurs in approximately 1 to 5 per 1000 live births and when permanent unilateral hearing loss is included, the incidence increases to 8 per 1000 live births [1-3].

The prevalence of hearing impairment is $1-8$ per 1000 newborns in India as documented by various studies using different screening protocols $[13,16,17]$. In a pilot study by Nagapoornima et al showed that screening only "at risk" newborns may miss up to $70 \%$ of newborns with hearing impairment. Of the 1490 not at-risk infants screened 7 had hearing impairment leading to an incidence of 4.70 per 1000 screened while in 279 at risk infants screened 3 had hearing impairment which is an incidence of approximately 10.75 per 1000 screened [16]. Among 798 babies without risk factors screened from tertiary care centre in Central India, 11.2 per 1000 had hearing impairment [18]. Of the 439 infants with no risk screened only one had hearing impairment that is approximately 2.27 per 1000 [13].

This low incidence when compared to our study (6.1 per thousand) is possibly due to high fall out rate of $44.4 \%$ in their study [13]. However, in a study from Cochin, out of 10,165 babies screened, the incidence of hearing loss in the high-risk group was 10.3 per 1000 and 0.98 per 1000 in the no risk group [17].

Previous studies have indicated hearing loss to be the most frequently occurring birth defect. It is necessary and high time to implement and incorporate universal neonatal screening in our country to secure normal, social and holistic development of the child by detecting hearing loss at birth and providing remedial services at the earliest. Updated evidence from various studies now show that infants who were diagnosed and received appropriate intervention before six months of age scored 20 to 40 percentile points higher on schoolrelated measures (language, social adjustment and behaviour) compared with hearing-impaired children who received intervention later on [11,19-22].

\section{Conclusions}

A two-staged TEOAE hearing screening can be feasible option as newborn hearing screening method, for early detection of hearing impairment in all major hospitals. Our finding indicates hearing loss to be one of the most frequently occurring birth defect. Universal hearing screening is the need of the hour to detect large number of hearing impaired in the magnanimous "no risk" newborn population in our country. This method of universal screening of newborn for detection of hearing impairment is simple, reliable and cost effective and can be successfully implemented in all tertiary care hospitals.

\section{What this study adds to existing knowledge?}

This study has shown that two-stage TEOAE hearing screening can be successfully implemented as newborn hearing screening method in a hospital setting. Universal newborn hearing screening using two-stage Transient Evoked Oto Acoustic Emissions (TEOAE) and confirmatory test by auditory brainstem response (ABR) at 3 months of life is a viable method for early identification of congenital hearing loss even in developing countries like India.

Contribution of Authors: VGM-concept and design of the study, data interpretation and manuscript writing, SKR-collection of the data and analysis, JK-manuscript writing and critical revision of the article.

Funding: Nil, Conflict of interest: None initiated, Perission from IRB: Yes

\section{References}

1. Mehra S, Eavey RD, Keamy DG Jr. The epidemiology of hearing impairment in the United States: newborns, children, and adolescents. Otolaryngol Head Neck Surg. 2009 Apr;140(4):461-72. doi: 10.1016/j. otohns.2008.12.022.

2. Stach BA, Ramachandran VS. Hearing disorders in children. In: Madell JR, Flexer $\mathrm{C}$ eds. Pediatric Audiology: Diagnosis, Technology, and Management. New York: Thieme Medical Publishers Inc;2008.P.3-12

3. Mason J A, Herrmann K R. Universal Infant Hearing Screening by Automated Auditory Brainstem Response Measurement. Pediatrics. 1998; 101(2):221-228.

4. Yoshinaga-Itano C, Sedey AL, Coulter DK, Mehl AL. Language of early- and later-identified children with hearing loss. Pediatrics. 1998 Nov;102(5):1161-71.

5. Norton SJ, Gorga MP, Widen JE, et al. Identification of neonatal hearing impairment: evaluation of transient evoked otoacoustic emission, distortion product otoacoustic emission, and auditory brain stem response test performance. Ear Hear. 2000 Oct;21(5):508-28. 
6. R. Keren, M. Helfand, C. Homer, H. McPhilips and T. A. Lieu, "Projected Cost Effectiveness of Statewide Univer- sal Newborn Hearing Screening. Pediatrics. 2002;110(5):855-864.

7. Early identification of hearing impairment in infants and young children. NIH Consens Statement. 1993 Mar $1-3 ; 11(1): 1-24$.

8. Joint Committee on Infant Hearing. Year 2007 position statement: principles and guidelines for early hearing detection and intervention programs. Pediatrics. 2007; 120(4):898-921.

9.www.who.int/pbd/deafness/world-hearing-day/WHD 2016_Brochure_EN_2.pdf.Accesed on $1^{\text {st }}$ october 2018.

10. American Academy of Pediatrics, Joint Committee on Infant Hearing Year 2007 position statement: Principles and guidelines for early hearing detection and intervention programs. Pediatrics. 2007; 120(4): 898-921.

11.Patel H, Feldman M. Universal newborn hearing screening. Paediatr Child Health. 2011 May; 16 (5): 301-10.

12. Canale A, Favero E, Lacilla $M$, et al. Age at diagnosis of deaf babies: a retrospective analysis highlighting the advantage of newborn hearing screening. Int J Pediatr Otorhinolaryngol. 2006 Jul; 70(7):1283-9. Epub 2006 Feb 20.

13. Rai N, Thakur N. Universal screening of newborns to detect hearing impairment--is it necessary? Int $\mathrm{J}$ Pediatr Otorhinolaryngol. 2013 Jun;77(6):1036-41. doi: 10.1016/j.ijporl.2013.04.006. Epub 2013 May 1.

14. Sangita M K, Patil H. Universal Screening in Newborn. Journal of medical science and clinical research. 2017;5(8):26396-26402.
Original Research Article

15. Augustine AM, Jana AK, Kuruvilla KA, et al. Neonatal hearing screening--experience from a tertiary care hospital in southern India. Indian Pediatr. 2014 Mar; 51(3):179-83. Epub 2013 Oct 5.

16. Nagapoornima P, Ramesh A, Srilakshmiet al. Universal hearing screening. Indian J Pediatr. 2007 Jun; 74 (6):545-9.

17. Paul AK. Early identification of hearing loss and centralized newborn hearing screening facility-the Cochin experience. Indian Pediatr. 2011 May;48 (5): $355-9$.

18. Anand S, Tiwari A, Goyal S. Prospective study for newborn hearing screening-A experience from tertiary care centre in central India. Int J Pediatr Res. 2016; 3(9):668-671.doi:10.17511/ijpr.2016.9.07

19. Nelson HD, Bougatsos C, Nygren P; et al. Universal newborn hearing screening: systematic review to update the 2001 US Preventive Services Task Force Recommendation. ediatrics. 2008 Jul;122(1):e266-76. doi: 10.1542/peds.2007-1422.

20. Korver AM, Konings S, Dekker FW, Beers M, Wever C C, Frijns J H M et al. Newborn hearing screening vs. later hearing screening and developmental outcomes in children with permanent childhood hearing impairment. JAMA. 2010;304(15):1701-1708.

21. McCann DC, Worsfold S, Law CM, Mullee M, Petrou S, Stevenson $\mathrm{J}$ et al. Reading and communication skills after universal newborn hearing screening for permanent childhood hearing loss. Arch Dis Child. 2009;94(4):293-297.doi:10.1136/adc.2008.151217

22. Yoshinaga-Itano C, Coulter D, Thomson V. The Colorado Newborn Hearing Screening Project: Effects on speech and language development for children with hearing loss. J Perinatol. 2000; 20 (8 Pt 2suppl): S132- S137.

\section{How to cite this article?}

Manjunath V.G, Reddy S.K, K. Jagadish Kumar. New born hearing screening programme at tertiary care hospital from South Karnataka-our experience. Int J Pediatr Res. 2018;5(10):527-531. doi:10.17511/ijpr.2018.i10.08. 